\title{
A Case study of cognitive and biophysical models of education as linked to anxiety and obsessive compulsive disorders
}

\author{
Kelly M. Maye
}

Abstract: Cognitive and biophysical factors have been considered contributors linked to identifiable markers of obsessive compulsive and anxiety disorders. Research demonstrates multiple causes and mixed results for the short-term success of educational programs designed to ameliorate problems that children with obsessive compulsive and anxiety disorders face in the day school setting. The consideration of cognitive and biophysical models of education as related to OCD and anxiety disorder has proven beneficial in determining appropriate treatment for the identified population of students. In this case study cognitive and biophysical factors are considered to address the referral, eligibility, placement, and treatment of a $4^{\text {th }}$ grade student named Ethan. Ethan exhibited OCD tendencies and elevated levels of anxiety. Ethan often responded to everyday situations with increased emotion of anger and worry, which presented an adverse impact on his educational performance. An array of information was collected as part of the special education evaluation conducted through several measures including: educational diagnostics, surveys, questionnaires, and interviews. Ethan was found eligible for services under the primary disability category "Emotional and Behavioral Disorder", related to diagnoses of Obsessive Compulsive and Anxiety Disorder. It was determined that the result of such chronic disorders limited Ethan's ability to access the educational environment, in the absence of specially designed instruction. Interventions considered applicable for implementation were Rational Emotive Behavior Therapy (REBT), as a form of psychotherapy, and Cognitive Therapy (CT).

Key words: Cognitive Model of Education, Biophysical Model of Education, Obsessive Compulsive Disorder, Anxiety, Disorder, Cognitive Therapy, Rational Emotive Behavior Therapy 


\section{Ethan}

\section{Student History}

Ethan is a ten year old, fourth grade, Caucasian male, who was referred for special education during his third grade school year. Information included in the study was obtained through the collection of school records, interviews, surveys, educational diagnostics, and observational data. More specifically, the Kaufman Assessment Battery for Children, Second Edition (KABC-II) was administered by the educational psychologist to determine his level of general intellect; the Kaufman Tests of Academic Achievement, Second Edition (KTEA-II) was administered by the educational diagnostician to determine academic levels of functioning; behavior observations and review of records was completed by the educational diagnostician, special educator, and educational psychologist; social developmental history was completed by the school psychologist and Ethan's parents; and parent, teacher, and physician questionnaires and surveys were completed to gather background information and information regarding probable areas of concern.

Ethan is an only child who lives with both his mother and father who reside in a mutual household and provide equal physical and emotional support to Ethan. Ethan lives in what seems to be a safe, inviting, and supportive environment. As interviews were typically conducted within the home environment, it was apparent that Ethan was happier and more at ease within the home environment than within the instructional environment. The home was always sanitary and of a welcoming nature. Ethan's bedroom was filled with maps and map activities, which was a high interest area for Ethan; he had a computer and computer desk that seemed to be set up for both leisure and school related activities. He seemed to rely heavily on his mother for homework support and to relay school related concerns. Ethan's household socioeconomic status would be considered middle to upper class, with his father employed at a prestigious marketing firm and his mother unemployed by choice. Both parents are educated and have received college/university level degrees.

Ethan's parents noted that although they did not recognize any significant cognitive delays early on, they did note a lack of noticeable vocabulary skills late within his second year of life. His parents also reported that he was very "standoffish" as a toddler and into early elementary age with his teachers and peers. He often disengaged in social play and group work. He was identified as systematic and unable to handle non-structured activities and change by his classroom teachers. It was also relayed by his parents that he never liked to get dirty, was a finicky eater, and liked order and sameness.

Per Ethan's original report, referencing a special education referral, he 
was originally referred due to recognizable deficits in the areas of mathematical reasoning and written expression. Concerns were also raised referencing Ethan's emotional instabilities and heightened levels of anxiety. After observational, interview, survey, and diagnostic data were compiled and analyzed, it was determined that Ethan exhibited signs of obsessive compulsive and anxiety disorders, with the persistent desire to be reassured and praised while seeking emotional support from his teachers and other adults in authoritative positions. Ethan's OCD and anxious behaviors concerned those around him in his home and school environments. His dependency on others to provide security and assistance, along with his need to control situations in an unrealistic manner, was additionally concerning to others who knew him on a personal level. Diagnostics confirmed typical development commensurate to his same aged peers referencing intelligence and achievement, yet cognitive efficiency and working memory were recognized as relative weaknesses. Diagnostic testing results also confirmed the existence of anxiety, OCD tendencies, and high achievement motivation. Ethan would often become very anxious and concerned referencing acceptance by others, peer relations, and personal academic performance. Furthermore, it can be assumed that Ethan's behavioral deficiencies adversely affected his academic performance.

\section{Two Models of Thought}

Two models of thought that support Ethan's deficiencies and the presence of a disability are the cognitive and biophysical models of learning. The cognitive model addresses Ethan's need to control and overanalyze situations in an unrealistic manner. His tendency to construe reality, as well as his obsessive compulsiveness lends consistency in support of the cognitive model. Academic deficits can also be defined through cognitive theory. The biophysical model, as related to Ethan's deficiencies, assumes the problem lies within and is medically related. Many theorists propose that environment, as perceived by the individual, can activate biological problems or genetic predispositions (Webber \& Plotts, 2008). It is possible that Ethan's anxiety was brought about by environmental factors within multiple settings, in which obsessive compulsive and anxious behaviors arise.

\section{Cognitive model of education}

Children, such as Ethan, with obsessive compulsive disorder often never stop worrying. OCD is an anxiety disorder that preoccupies one's thoughts and beliefs. The anxiety and worry is often so overbearing that children feel 
the need to repeat specific behaviors over and over again in hopes that everything will work out to their desire (The Nemours Foundation, 2010). Ethan consistently over obsessed about the future, and exhibited a strong need to control situations that are often beyond his control. When things go differently than expected, he became anxious and exhibited negative behaviors, which in return affected his academic performance. Positively noted; obsessive compulsive disorder is believed to be a disorder than can be cured, under the notion that "cognitions are under the control of the individual"; therefore can be altered (Webber \& Plotts, 2008, p.136). The cognitive model enforces the belief that people can change their thoughts and perceptions through means of self-control and self-regulation in pursuit of increasing mental stability and sustaining more positive and self-fulfilling behaviors. The biophysical model supports positive environmental change, or change of setting, that can positively alter student behaviors through effective intervention.

To understand the etiology and development of Ethan's disorder in analyzing cognitive processes, it is important to relay that his long-term unconscious cognitive processes are affected, as his irrational belief system and skewed perceptive thinking affects short-term processes; in his case by means of appraisals and expectations. Beck (1967) affirmed that such short-term processes can contribute to elevated levels of anxiety and worry (as cited in Webber \& Plotts, 2008), which was notably present in Ethan's situation. Individuals like Ethan, who are anxious, often expect negative things to occur. The repeated cycle of forming expectations often concludes false beliefs and alters the way one sees the world.

Appraisals, as the concept of individuals placing assumptions to beliefs, quite often affected Ethan's daily life. He frequently obtained a mentality, scientifically denoted, that one variable is always dependent on another; he overly obsessed on the outcome of multiple events with persistence. If he perceived the outcome inaccurately he may experience disappointment and dysfunctional feelings which would commonly lead to unhealthy behaviors. Beck's cognitive theory model expressed the idea that individuals who misinterpret facts and experiences in an atypical manner, limits their overall focus on negatively related concepts and forms misconceptions about future events (Allen, 2003). It is important for Ethan to set rational self-focused goals to aid in developing accurate assumptions and perceptions of current and future life events.

In relation to learning difficulties that Ethan experienced, cognitive theorists engage in the idea that information is learned through making connections; furthermore, linking prior knowledge to newly learned concepts. Grow (1996) suggested using elaboration as a positive strategy to enhance cogni- 
tion; he defined elaboration as linking prior knowledge to new ideas, so that the two may become more deeply intertwined, thus more commonly practiced. "Learning takes place when new information becomes part of existing knowledge" (Grow, 1996, p.3), yet in Ethan's case cognitive perceptions of life must be addressed prior to enforcing academic strategy, as a means to control behaviors. These behaviors often greatly impacted his overall academic performance and learning experiences. Grow (1996) affirmed that "knowledge can be called useful only if you can access it under appropriate circumstances" (p. 3). Ethan often allowed his knowledge of life concepts to guide his perceptions and appraisals, thus altering his expectations of himself and others. Oliver (1980) suggested that early propositions are linked to student satisfaction and more positive expectations, and that satisfaction increases as performance level increases. Ethan's situation supports the view that behavior can impede one's ability to perform. Students with conduct related disorders statistically perform poorly academically. Webber and Plotts (2008) claimed that students with conduct related disorders often exhibit impulsive behaviors, distractibility, and inattentiveness, which can easily lead to problems with self-control that may predispose them to academic related difficulty.

Rational emotive behavioral therapy (REBT) is a developed cognitive method that refers to changing one's belief system in order to cure disorders that relate to misperceptions and faulty belief systems (Webber \& Plotts, 2008). A prime example of Ethan's irrational behavior that may render the need for therapeutic intervention, such as REBT, is his constant worry and obsession in planning his future, as reported by his mother, which contributed to the irrational belief that if he doesn't start planning now, he will become a failure later. When children establish irrational beliefs, increased levels of anxiety can arise and the feeling of extreme discouragement can come about. Horowitz (2009) identified that the "mismatch between ability, expectations, and outcomes can cause tremendous disappointment and frustration, resulting in a cascade of emotions and behaviors that can interfere with everyday functioning" (p. 1).

\section{Biophysical model of education}

The biophysical model of education is a model that justifies disordered behavior as genetically, bio-chemically or neurologically related, as well as conceptualized through innate temperament (Webber \& Plotts, 2008). Research on genetic, biochemical, and neurological factors is linked to severe emotional and behavioral impairments. Rhodes and Tracy (1974) identified the biophysical model as a conceptual model that aids in under- 
standing emotional and behavioral disorders (as cited in Zabel, 1988). Temperamental and possible genetic factors may define Ethan's atypical behaviors, yet the idea that biochemical factors may contribute directly to his specific behavioral deficits was not addressed. Webber and Plotts (2008) informed that "temperament refers to a behavioral style that is an inborn tendency, yet is highly influenced by one's environment or setting" (p.93). Ethan exhibited four of the nine characteristics of temperament; defined by Thomas, Chess, and Birch (1969); including adaptability, threshold of responsiveness, intensity of reaction, and mood quality (as cited in Webber \& Plotts, 2008). Temperament factors related to EBD describes inborn tendencies to react with inappropriate, disruptive, and negative behaviors, yet is also influenced by environmental factors. Rothbart and Mauro (1990) related temperament to "activity level, rhythmicity, fearful distress, positive affect, attention span and persistence, and irritability and distress" that is exhibited over a period of time and is influenced by environmental factors (as cited in Webber \& Plotts, 2008, p.93). An abundance of educating professionals and researchers acknowledge that innate temperamental factors do often define an emotional and/or behavioral disorder, yet particular mental patterns cannot be determined when referencing the diagnosis of EBD, due to the existence of concomitant behaviors.

Implications taken together with the complex nature of defining biophysical factors pose distinct challenges for educators at all levels of education (Tibell \& Rundgren, 2009). Genetic factors refer to inherited disorders, such as manic depression, anxiety, mood disorder, and anti-social personalities, which are suspected genetically transmitted disorders that may be linked to exhibited signs of emotional and/or behavioral disturbance (Webber \& Plotts, 2008). A mass of educating professionals support the idea that an emotional or behaviorally related disorder can be generated within the individual through genetic factors, and that genetically determined factors can be changed over time through environmental cause (Webber \& Plotts, 2008). Evidence has pinpointed genetics as a predisposing factor of obsessive compulsive disorder; Beck (1976) claimed that many people with OCD have one or more family members who also have the disorder or other anxiety disorders influenced by the brain's serotonin levels (as cited in Allen, 2003). "Scientists have come to believe that the tendency, or predisposition, for someone to develop the serotonin imbalance that contributes to obsessive compulsive tendencies is often genetically inherited" (The Nemours Foundation, 2010, p. 2). Obsessive compulsive disorder and mood disorders, in which Ethan has exhibited signs of, correlate with elevated levels of neurotransmitters, specifically serotonin that is present in the blood system and contributes to heightened anxiety and stress. 
Although it is largely agreed upon that biophysical factors do contribute to emotional and behavioral deficiencies; defining and validating the existence of such factors may require attention from external sources. The biophysical model supports underlying factors that often contribute to the diagnosis of EBD, but not legitimately through expertise of educating professionals or public school related staff. A school psychologist can offer valuable insight on underlying factors obtained through evaluation processes, yet typically the identification and noted presence of biophysical attributes is established by medical personnel. "In the biophysical model, medical interventions are sought when a physiological cause for emotional and/or behavioral disorder is hypothesized or identified" (Webber \& Plotts, 2008, p.97). Educating professionals, notably in the field of special education, are bound to addressing deficiencies through the process of intervention and productive teaching. Applications of genetic counseling and technologies may be productive in directing a possible genetic etiological factor, as applications of nutrition therapy, counseling and behavioral interventions may be appropriate in addressing a possible etiological factor of temperament (Webber \& Plotts, 2008). Webber and Plotts (2008) suggested the use of developmental histories and genetic testing to determine the existence of genetic related disabilities, and the administering of temperament assessment and DNA testing to determine the presence of temperament as an etiological factor related to possible biophysical deficiencies.

\section{Conclusions}

As the referral process was concluded, Ethan was found eligible for services under the primary disability category "Emotional and Behavioral Disorder", related to diagnoses of Obsessive Compulsive and Anxiety Disorder. It was determined that the result of such chronic disorders limited Ethan's ability to access the educational environment, in the absence of specially designed instruction.

\section{Treatment}

Interventions considered applicable for implementation were Rational Emotive Behavior Therapy (REBT), as a form of psychotherapy, and Cognitive Therapy (CT). It was determined that REBT would be administered through pull out services, by the educational psychologist, during the regular instructional day. REBT is to be structured in an active-directive manner, with the goal of working through a set of target problems and establishing therapeutic goals. Over time, Ethan is expected to work through his 
problems and learn to generalize insights to other relevant, more positive situations. The outlook is to utilize REBT as an intervention with brief and limited measure. The goal is for Ethan to transition from REBT with a developed understanding of how to deal with the elusive condition of OCD and accept emotional responsibility for his problems, while obtaining the willingness and determination to change targeted behaviors.

Cognitive Therapy (CT) is to be implemented both during and after REBT has been concluded. The goal of using CT with Ethan to concentrate on the obsessions and compulsions is to intertwine cognitive conceptualization and management. Cognitive conceptualization should be implemented in the absence of challenge, as the focus is reassurance oriented. "Helping people to separate themselves (i.e. their "genuine" identity) from the emotional and/or moral implications of what this disorder seems to represent, is a major portion of cognitive conceptualization" (Phillipson, 2011, p. 2). Cognitive management, as the latter approach, is to be implemented and reinforced in both the therapeutic setting and instructional setting with Ethan. Cognitive management can be used to help Ethan respond effectively to cognitive prompts or physiological experiences of perceived danger or worry. Phillipson (2011) conferred how fundamental it is that one's scheme of creating cognitive restoration not be a pre-programmed, rote, automatic reaction. The more one inculcates an authentic poignant importance into the responses, the more they will boost the effectiveness of the therapy (Reed, 2010). Removal of variables that reinforce the behavior, habitually revisiting areas that produce anxiety and obsessions as a means to reduce Ethan's brain sensitivity to particular emotions, and exposing him to stressors that engage ritual, will work together in hopes of creating increased resilience to reversion.

\section{References}

Allen, J. P. (2003). An overview of Beck's cognitive theory of depression in contemporary literature [Unpublished works]. Rochester Institute of Technology.

Beck, A. T. (1976). Cognitive therapy and emotional disorders. New York, NY: International University Press.

Grow, G. (1996). Serving the strategic reader: Cognitive reading theory and its implications for the teaching of writing [Unpublished works]. Florida A\&M University.

Horotwitz, S. H. (2009). Behavior problems and learning disabilities. National Center for Learning Disabilities. Retrieved April 16, 2012, from http://www.ncld.org/ldbasics/ld-aamp-social-skills/social-aamp-emotionalchallenges/behavior-problemsand-learning-disabilities.

Oliver, R. L. (1980). A cognitive model of the antecedents and consequences of satisfaction decisions. Journal of Marketing Research, 17(4), 460-469. 
Phillipson, S. (2011). When seeing is not believing: A cognitive therapeutic differentiationbetween conceptualizing and managing OCD. Retrieved April, 16, 2012, from http:/ / www.ocdonline.com/definecbt.php

Reed, S. K. (2010). Cognition: Theories and applications ( $8^{\text {th }}$ ed.). Belmont, CA: Wadsworth,Cengage Learning.

The Nemours Foundation. (2011). Obsessive-compulsive disorder. Retrieved April, 16, 2012, from http://kidshealth.org/kid/feeling/emotion/ocd.html\#

Thomas, A., Chess, S. \& Birch, H. (1969). Temperament and behavior disorders in children.New York, NY: University Press.

Tibell, L., A., E. \& Rundgren, C. J. (2009). Characteristics and implications for education and research. Manuscript submitted for publication, Department of Thematic Studies, Linkoping University, Norrkoping, Sweden.

Webber, J. \& Plotts, C.A. (2008). Emotional and behavioral disorders: Theory and practice $\left(5^{\text {th }}\right.$ ed.). Boston, MA: Pearson Education, Inc.

Zabel, R. H. (1988). Use of time by teachers of behaviorally disordered students: A replication. Behavioral Disorders, 13(2), 89-97.

\section{Author:}

Kelly M. Maye, Ph.D. candidate

Carter G. Woodson Academy

Faculty Special Education

1813 Charleston Drive

Lexington

Kentucky

40505

USA

Email: kmaye011@odu.edu 